\title{
Constraints on Metastable Helium in the Atmospheres of WASP-69b and WASP-52b with Ultranarrowband Photometry
}

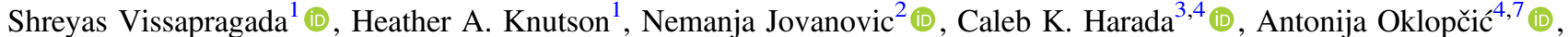 \\ James Eriksen $^{5}$, Dimitri Mawet ${ }^{2,6}$ (D), Maxwell A. Millar-Blanchaer ${ }^{2}$ (1), Samaporn Tinyanont ${ }^{2}$ (10), and Gautam Vasisht ${ }^{6}$ (1) \\ ${ }^{1}$ Division of Geological and Planetary Sciences, California Institute of Technology, 1200 East California Blvd, Pasadena, CA 91125, USA; svissapr@caltech.edu \\ ${ }^{2}$ Department of Astronomy, California Institute of Technology, 1200 East California Blvd, Pasadena, CA 91125, USA \\ ${ }^{3}$ Department of Astronomy, University of Maryland, 4296 Stadium Drive, College Park, MD 20742, USA \\ ${ }^{4}$ Center for Astrophysics | Harvard \& Smithsonian, 60 Garden Street, MS-16, Cambridge, MA 02138, USA \\ ${ }^{5}$ Palomar Observatory, California Institute of Technology, 35899 Canfield Rd, Palomar Mountain, CA 92060, USA \\ ${ }^{6}$ Jet Propulsion Laboratory, California Institute of Technology, 4800 Oak Grove Dr, Pasadena, CA 91109, USA \\ Received 2020 February 4; revised 2020 April 24; accepted 2020 April 27; published 2020 May 28
}

\begin{abstract}
Infrared observations of metastable $2^{3} \mathrm{~S}$ helium absorption with ground- and space-based spectroscopy are rapidly maturing, as this species is a unique probe of exoplanet atmospheres. Specifically, the transit depth in the triplet feature (with vacuum wavelengths near $1083.3 \mathrm{~nm}$ ) can be used to constrain the temperature and mass-loss rate of an exoplanet's upper atmosphere. Here, we present a new photometric technique to measure metastable $2^{3} \mathrm{~S}$ helium absorption using an ultranarrowband filter (FWHM $0.635 \mathrm{~nm}$ ) coupled to a beam-shaping diffuser installed in the Wide-field Infrared Camera on the 200 inch Hale Telescope at Palomar Observatory. We use telluric $\mathrm{OH}$ lines and a helium arc lamp to characterize refractive effects through the filter and to confirm our understanding of the filter transmission profile. We benchmark our new technique by observing a transit of WASP-69b and detect an excess absorption of $0.498 \% \pm 0.045 \%(11.1 \sigma)$, consistent with previous measurements after considering our bandpass. We then use this method to study the inflated gas giant WASP-52b and place a 95th percentile upper limit on excess absorption in our helium bandpass of $0.47 \%$. Using an atmospheric escape model, we constrain the massloss rate for WASP-69b to be $5.25_{-0.46}^{+0.65} \times 10^{-4} M_{\mathrm{J}} \mathrm{Gyr}^{-1}\left(3.32_{-0.56}^{+0.67} \times 10^{-3} \mathrm{M}_{\mathrm{J}} \mathrm{Gyr}^{-1}\right)$ at $7000 \mathrm{~K}(12,000 \mathrm{~K})$. Additionally, we set an upper limit on the mass-loss rate of WASP-52b at these temperatures of $2.1 \times 10^{-4} M_{\mathrm{J}} \mathrm{Gyr}^{-1}\left(2.1 \times 10^{-3} M_{\mathrm{J}} \mathrm{Gyr}^{-1}\right)$. These results show that ultranarrowband photometry can reliably quantify absorption in the metastable helium feature.
\end{abstract}

Unified Astronomy Thesaurus concepts: Exoplanet astronomy (486); Exoplanet atmospheres (487); Planetary atmospheres (1244); Transmission spectroscopy (2133); Infrared photometry (792); Narrow band photometry (1088)

Supporting material: data behind figures

\section{Introduction}

Many of the currently known exoplanets are on short-period orbits and thus experience severe insolation. Such extreme environments can radically alter planetary evolution, potentially driving atmospheric mass loss via thermal escape (e.g., Tian 2015; Owen 2019). Mass loss can in turn leave substantial imprints on observed planetary statistics, such as the dearth of planets between 1.5 and 2 Earth radii (the "radius gap" or "evaporation valley") and the so-called "Neptune desert" in the radius-period plane (Lopez \& Fortney 2013; Owen \& Wu 2013, 2017; Fulton et al. 2017; Fulton \& Petigura 2018; van Eylen et al. 2018; Cloutier \& Menou 2020; HardegreeUllman et al. 2020). Over the past two decades, most measurements of mass-loss rates for close-in planets have been conducted at ultraviolet wavelengths, with Ly $\alpha$ detections for HD 209458b (Vidal-Madjar et al. 2003), HD 189733b (Lecavelier Des Etangs et al. 2010; Lecavelier des Etangs et al. 2012), GJ 436b (Kulow et al. 2014; Ehrenreich et al. 2015; Lavie et al. 2017), and GJ 3470b (Bourrier et al. 2018); tentative/marginal signals for TRAPPIST-1b and c (Bourrier et al. 2017a), Kepler-444e and f (Bourrier et al. 2017b), and K2-18b (dos Santos et al. 2020); and nondetections for $55 \mathrm{Cnc}$

\footnotetext{
${ }^{7}$ NHFP Sagan Fellow.
}

e (Ehrenreich et al. 2012), HD 97658b (Bourrier et al. 2017c), GJ 1132 b (Waalkes et al. 2019), and $\pi$ Men c (García Muñoz et al. 2020). While in theory the large cross section of this line should result in strong absorption during exoplanet transits, in practice geocoronal emission and interstellar absorption effectively mask out the line core for most stars, requiring these studies to study the absorption in the line's extended wings.

The neutral helium triplet (with vacuum wavelengths near $1083.3 \mathrm{~nm}$ ) offers a way to circumvent the limitations of $\mathrm{Ly} \alpha$ observations (Seager \& Sasselov 2000; Oklopčić \& Hirata 2018) by shifting to infrared wavelengths where both the Earth's atmosphere and the interstellar medium (e.g., Indriolo et al. 2009) are effectively transmissive. Spake et al. (2018) were the first to successfully observe an enhanced transit depth in He I for WASP107b with Wide Field Camera 3 (WFC3) on the Hubble Space Telescope (HST). Soon after, ground-based observations with the CARMENES high-resolution $(R \sim 80,000)$ spectrograph on the $3.5 \mathrm{~m}$ telescope at Calar Alto Observatory have confirmed the absorption signal and measured the He I line shape for HAT-P11b (Allart et al. 2018) and WASP-107b (Allart et al. 2019), and have additionally revealed excess helium absorption signals for HD 189733b (Salz et al. 2018), HD 209458b (Alonso-Floriano et al. 2019), and WASP-69b (Nortmann et al. 2018). HST WFC3 observations were also used to identify He I absorption for 
HAT-P-11b (Mansfield et al. 2018), and recently Keck II/ NIRSPEC and the Habitable-zone Planet Finder have observed helium in the atmospheres of WASP-107b (Kirk et al. 2020) and GJ 3470b (Ninan et al. 2020), respectively. We note also the reported nondetections of helium in the atmospheres of KELT-9b, GJ 436b (both Nortmann et al. 2018), WASP-12b (Kreidberg \& Oklopčić 2018), GJ 1214b (Crossfield et al. 2019), and K2-100b (Gaidos et al. 2020). Due to its observational accessibility for ground- and space-based facilities, the helium triplet has been firmly established as a window into the upper atmospheres of exoplanets.

Here, we introduce ultranarrowband helium photometry, a ground-based technique complementary to high-resolution spectroscopy that is specifically crafted to measure the helium absorption depth using an ultranarrow bandpass filter. In this work, we benchmark our new technique on the Wide-field Infrared Camera (WIRC), at the prime focus of the Hale 200" telescope at Palomar Observatory. We first measure the He I light curve of WASP-69b, a $1000 \mathrm{~K}$, Saturn-mass, and Jupitersized planet orbiting a K5 host star with $J=8$ (Anderson et al. 2014). We compare our results to those of Nortmann et al. (2018), and show that our results agree well with theirs. We then present the first He I light curve of the slightly warmer $(1300 \mathrm{~K})$, larger $\left(1.27 R_{\mathrm{J}}\right)$, and heavier $\left(0.46 M_{\mathrm{J}}\right)$ planet WASP$52 \mathrm{~b}$, which orbits a K2 host star with $J=10.5$ (Hébrard et al. 2013). In Section 2, we detail the experimental design of our ultranarrowband helium photometer. We discuss our observations and data reduction techniques in Section 3. We present our results in Section 4, and conclude with a look toward future applications of ultranarrowband photometry in Section 5.

\section{Experimental Design}

Our experiment is analogous to broadband transit photometry performed previously (Vissapragada et al. 2020) with WIRC (Wilson et al. 2003) on the Hale 200" telescope at Palomar Observatory. The sole difference is that we use an ultranarrowband filter (manufactured by Alluxa) that is centered on the helium feature. We used a combination of identifiable telluric $\mathrm{OH}$ emission lines as well as a helium lamp (naturally producing the feature in emission) to calibrate out refractive effects and ensure our knowledge of the filter transmission profile is accurate.

\subsection{Filter Properties}

Specifically, our filter has a center wavelength of $1083.3 \mathrm{~nm}$ in vacuum, at $77 \mathrm{~K}$, and at an angle of incidence (AOI) of $7^{\circ}$; an FWHM of $0.635 \mathrm{~nm}$; and a maximum transmission of $95.6 \%$ (averaged across five positions on the filter). To cover the full spectral range to which our $2.5 \mu \mathrm{m}$ cutoff Hawaii-II detector is sensitive, the filter also has OD4 absolute out-ofband blocking (i.e., a transmission less than $0.01 \%$ everywhere outside the passband) from $500-3000 \mathrm{~nm}$. We additionally utilize an engineered diffuser (located in a separate filter wheel from the helium filter) that molds the stellar point-spread functions (PSFs) into a top-hat shape with a FWHM of $3^{\prime \prime}$. The diffuser increases observing efficiency and limits systematics related to PSF variations. When combined with our guiding software, which can keep pointing stable to within 2-3 pixels (equivalent to 0 ". 5-0." 75) over an entire night, this setup allows for powerful control of time-correlated systematics (Stefansson et al. 2017). With this setup in place, we have recently demonstrated a precision of $0.16 \%$ per 10 minute bin for $J=14$ mag stars (Vissapragada et al. 2020).

Consideration of refractive effects is critical for such a narrowband filter, especially with a wide-field camera (e.g., Ghinassi et al. 2002; Tinyanont et al. 2019). Critically, the filter wheels in WIRC are fixed at a $7^{\circ}$ tilt to minimize ghosting (Wilson et al. 2003), and the filters cannot be angle-tuned. Because most rays forming the image encounter the filter at nonnormal incidence due to the filter tilt (as well as the diversity of angles for each field point), they experience a different passband. As a result, different positions on the detector correspond to different filter transmission profiles. While this effect is noticeable even for broadband filters (Ghinassi et al. 2002; Tinyanont et al. 2019), the amplitude of the shift in wavelength space is small compared to the width of the bandpass, and thus it is typically ignored without consequence. For ultranarrowband filters however, this shift can easily be larger than the bandwidth of the filter itself (e.g., Baker et al. 2019). The success of our experiment therefore depended largely on the success of our wavelength calibration.

\subsection{Wavelength Calibration with Telluric $\mathrm{OH}$ Lines}

To begin calibrating refractive effects, we used known telluric emission lines in the sky background to construct a model for the position-dependent wavelength shift. We used a sky background frame (constructed with a four-point dither near WASP-69) shown in Figure 1(a). Rays that pass through the filter at the same angle of incidence trace out semicircular arcs across the detector, and telluric $\mathrm{OH}$ emission lines thus appear as bright arcs on the detector. The offset center of the circles toward the top of the image is due to the aforementioned $7^{\circ}$ tilt of the filter wheel; if the filter wheel was not tilted, the circles would be centered on the detector (see, e.g., Sing et al. 2011). Instead, the center of the circle to which the arcs belong is the "zero-point" of the filter; i.e., where rays encounter the filter at normal incidence. The best-fitting circular arcs to the emission features give the detector position for the zero-point: $\left(x_{0}, y_{0}\right)=(1037,2120)$, where the origin of the coordinate system is the bottom left corner of the image. The angle of incidence on the filter at detector position $(x, y)$ can be written as a function of the radial distance from the zero-point $r=\sqrt{\left(x-x_{0}\right)^{2}+\left(y-y_{0}\right)^{2}}$ :

$$
\begin{aligned}
\theta(r) & =(\text { pixel scale })(\text { magnification }) r \\
& =(0 . \prime 25 / \mathrm{px})\left(\frac{5.08 \mathrm{~m}}{5.2364 \times 10^{-2} \mathrm{~m}}\right) r \\
& =\left(24 !^{\prime \prime} 3 / \mathrm{px}\right) r
\end{aligned}
$$

where the magnification is calculated as the primary mirror diameter over the beam diameter. By extracting the median count value in radial steps outward from the zero-point, we construct a spectrum of the sky. To convert the spectrum into more useful wavelength units, we note that the $\mathrm{OH}$ emission lines in the image can be individually identified as $Q$ and $R$ branch lines from the $\nu=5-2$ band for ground-state $\left(\mathrm{X}^{2} \Pi\right)$ OH (Bernath \& Colin 2009; Oliva et al. 2015). Using the known wavelengths of these lines, we can fit to the equation for wavelength shift as a function of angle of incidence $\theta$ (e.g., 


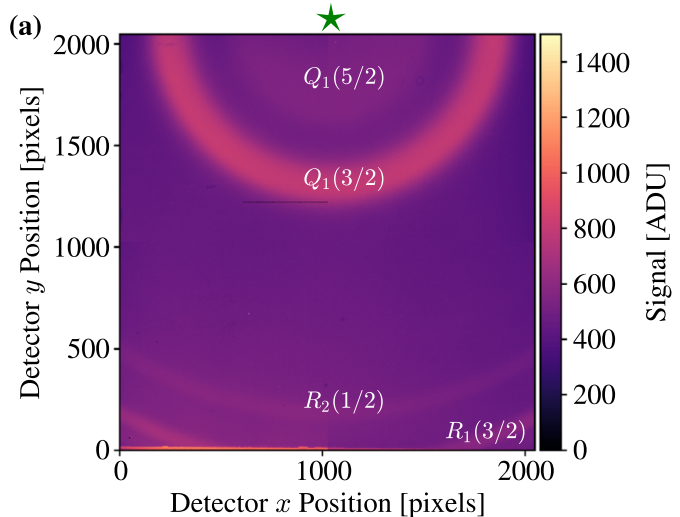

(b)

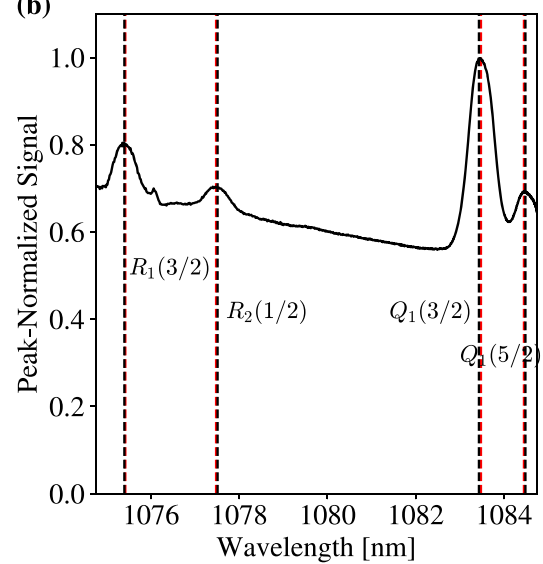

(c)

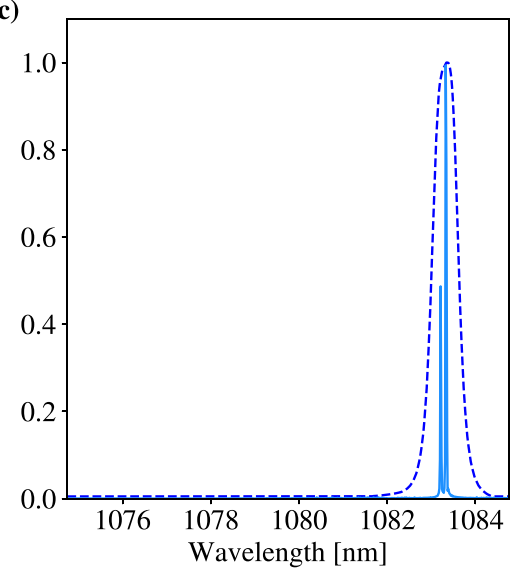

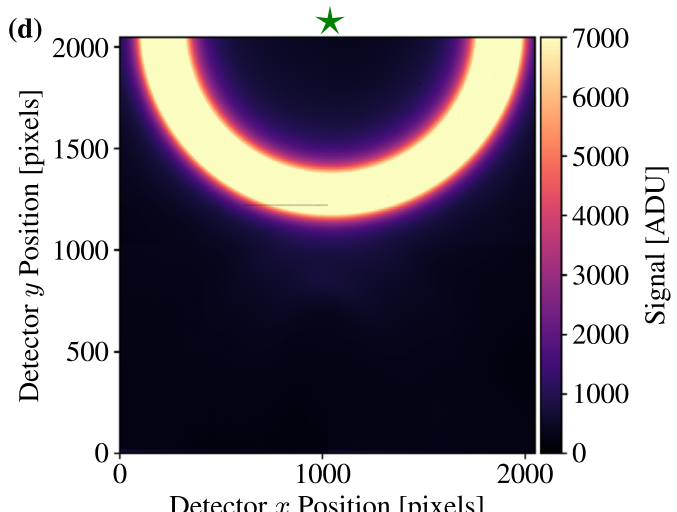

Detector $x$ Position [pixels]

(e)

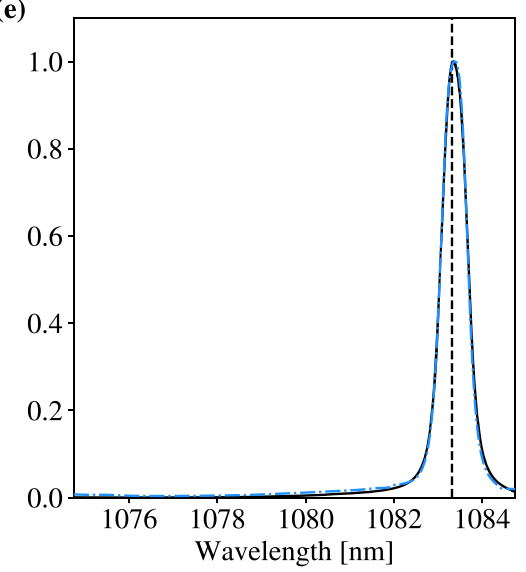

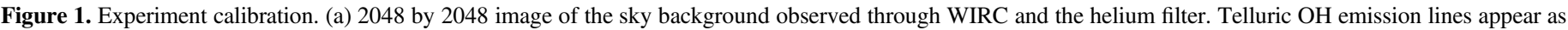

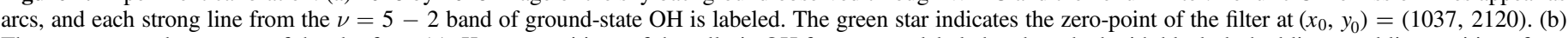

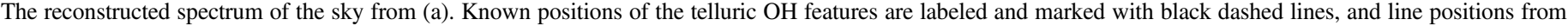

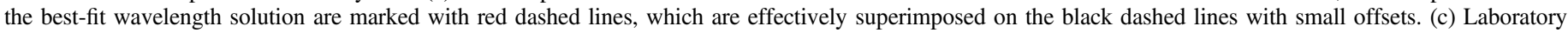

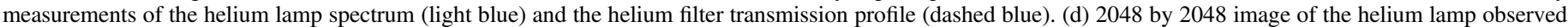

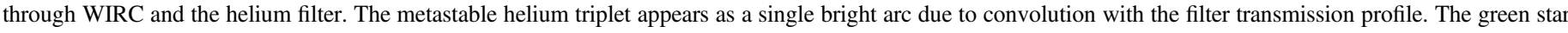

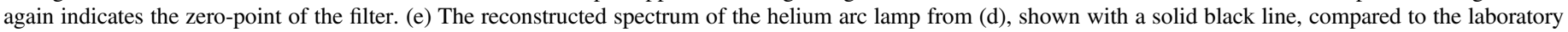
spectrum of the helium arc lamp convolved with the filter transmission profile (dashed light blue) and the known wavelength of the feature (dashed black).

(The data used to create this figure are available.)

Ghinassi et al. 2002):

$$
\lambda(\theta)=\lambda_{0} \sqrt{1-\frac{\sin ^{2}(\theta)}{n_{\mathrm{eff}}^{2}}},
$$

where $\lambda_{0}$ is the central wavelength of the filter at normal incidence, and $n_{\text {eff }}$ is the effective index of refraction for the filter. A nonlinear least-squares fit to the known wavelengths of the telluric lines gives $\lambda_{0}=1084.80 \mathrm{~nm}$ and $n_{\mathrm{eff}}=1.948$. Combined with Equation (1), this fully specifies the wavelength solution for every pixel on the detector as a function of the distance $r$ from $\left(x_{0}, y_{0}\right)=(1037,2120)$. The spectrum of the sky background constructed with this transformation is given in Figure 1(b).

\subsection{Helium Arc Lamp Calibration}

We used a helium arc lamp, which is a natural source of the He I triplet in vacuum, to confirm our wavelength solution and test our knowledge of the filter transmission profile. First, we measured the spectrum of the arc lamp and the transmission spectrum of the helium filter (backlit by white light) using an optical spectrum analyzer (OSA; ThorLabs \#OSA202C). The
OSA uses Fourier transform spectroscopy to deliver laboratory spectra at high resolving power $(R \sim 75,000)$. We show the laboratory spectra in Figure 1(c), where the two-component structure of the helium feature is clear (the two lines on the red side of the triplet are blended even at this resolution).

We then installed the helium arc lamp at the Hale 200" and used it to uniformly illuminate the region of the dome normally used for flat fields. When the helium lamp is observed through WIRC, the resultant bright arc (Figure 1(d)) is where the filter transmission profile maximally overlaps with the triplet helium feature, so during science observations we place the target within the region delineated by this arc. In practice, we take an arc lamp calibration frame before each observation, and we move the target star to a spot with a count level within $5 \%$ of the peak counts in the calibration frame. Since there is a semicircular locus on the detector that satisfies this criterion, the exact location is selected during observations to optimize the number of reference stars and avoid detector regions with many bad pixels or defects. Using the same procedure as detailed in Section 2.2, we extract the spectrum from the image in Figure 1(d), and use the wavelength solution from Equation (2) to convert from AOI to nanometers. The resulting spectrum (Figure 1(e)) peaks at $1083.3 \mathrm{~nm}$, indicating that our 
(a)
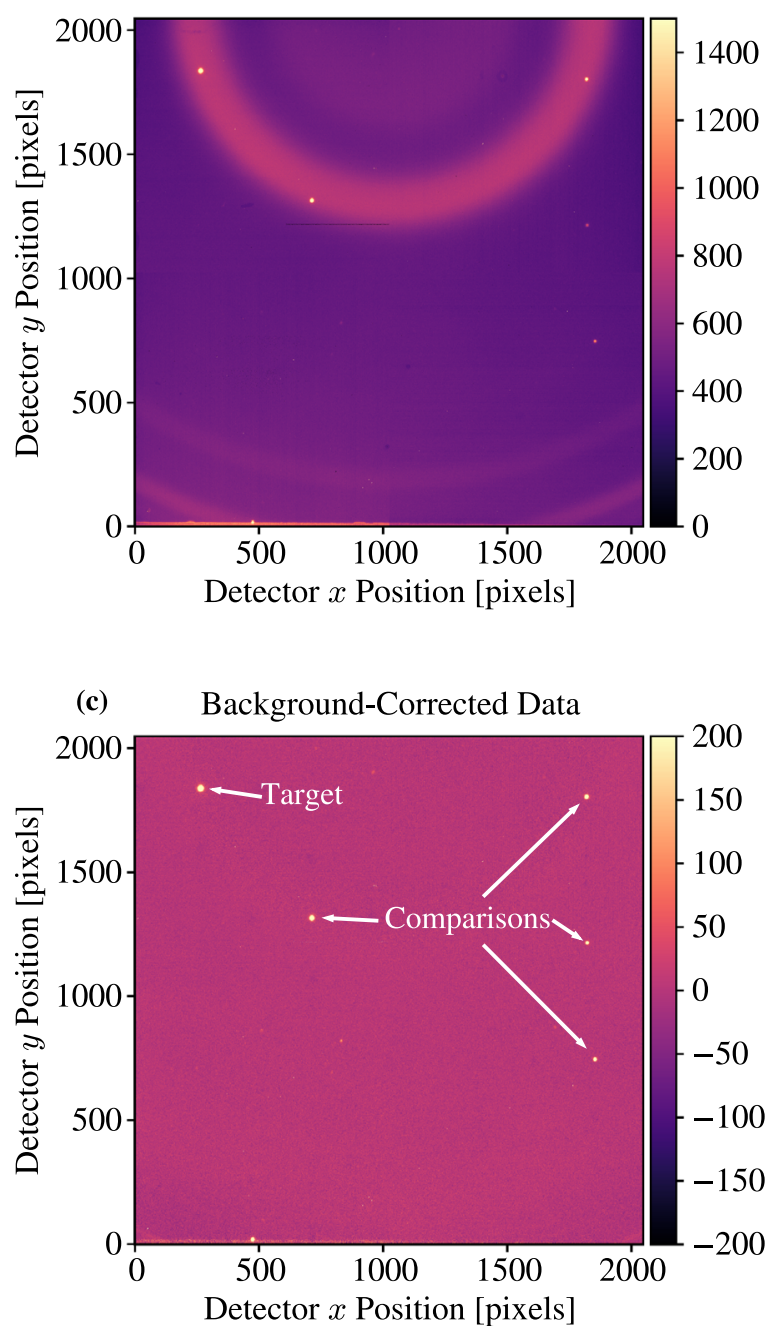

(b)

Sky Background
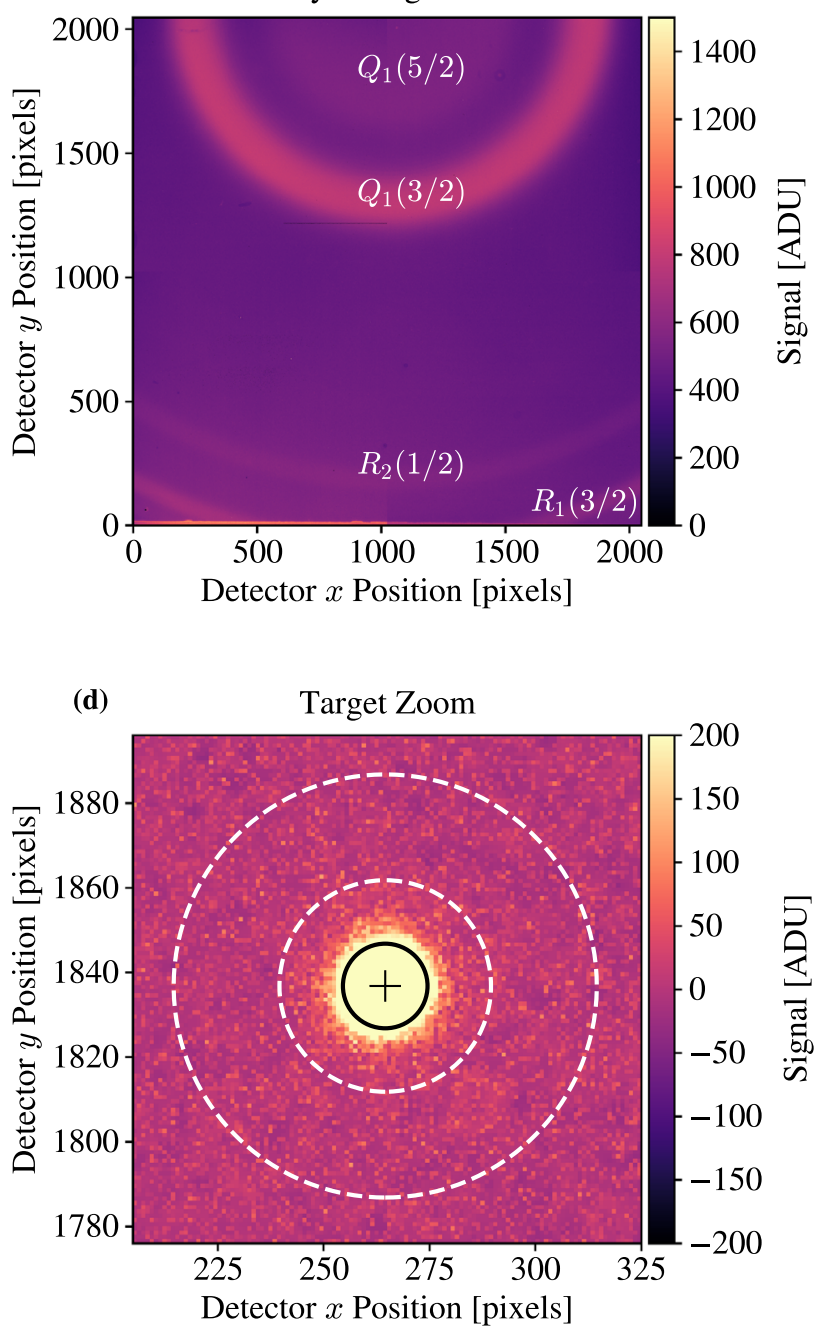

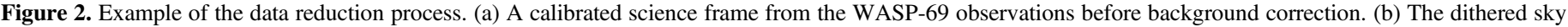

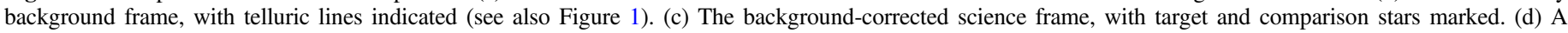

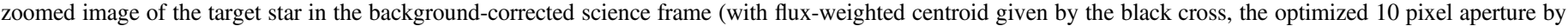
the black circle, and the annulus used for residual background estimation by the white dashed circles).

empirical wavelength solution correctly predicts the location of the helium triplet as measured by the lamp observation. Finally, as a test of the filter transmission profile, we convolve the laboratory measurements of the helium feature and the filter transmission profile, and overplot the result on the WIRC spectrum in Figure 1(e). The laboratory measurements (dotteddashed blue curve) and observations (black curve) show very good agreement.

\section{Observations}

\subsection{Data Collection}

We observed WASP-69b through our helium filter and beam-shaping diffuser on 2019 August 16 (UT), and we observed WASP-52b with the same setup on 2019 September 17 (UT). Before beginning both science observations, we constructed a sky background frame with a simple four-point dither. Images in the dither sequence were first sigma-clipped to remove the sources, then median-scaled to the first image in the stack, and finally median stacked to produce the sky background frame. We then collected science data, choosing exposure times to keep the maximum count level for the sources and comparison stars ( $\sim 12,000$ ADU) well within the linearity regime for our detector while maintaining a good observing efficiency. For WASP-69b, we collected science data from UT 04:26:06 to 11:00:00 with an exposure time of $60 \mathrm{~s}$; our observations began at airmass 1.73 , reached a minimum airmass of 1.28, and then rose again until we stopped collecting data at airmass 2.49. For WASP-52b, we collected science data from UT 03:16:57 to 11:14:49 with an exposure time of $90 \mathrm{~s}$; our observations began at airmass 2.04 , reached a minimum airmass of 1.10, and then rose again until we stopped collecting data at airmass 1.96 .

\subsection{Data Reduction \\ 3.2.1. Image Calibration}

We show an example science image for WASP-69b in Figure 2. All science data were dark-subtracted and flat-fielded, and during this procedure bad pixels were flagged and corrected using the process described by Tinyanont et al. (2019) and Vissapragada et al. (2020). Unlike the case in Vissapragada et al. (2020), however, the background is not uniform across the detector. Contamination from telluric $\mathrm{OH}$ 
emission is clearly visible, but because these lines have a very unique spatial structure their contribution can be identified and removed. Presently, we do not correct for telluric water during image calibration. We note that the water line at $1083.507 \mathrm{~nm}$ (vacuum wavelength in the observer rest frame) can potentially affect the observations, though it is diluted by a minimum of $\sim 20 \%$ by the filter transmission at the target position. This line does not encroach upon the helium triplet unless the triplet is redshifted by $48.7 \mathrm{~km} \mathrm{~s}^{-1}<v<83.6 \mathrm{~km} \mathrm{~s}^{-1}$ relative to the observer. This does not occur for WASP-69b and WASP-52b (and in fact we do not observe targets at such velocity shifts because the helium signal would be spatially shifted from the positions set by the calibration lamp) so our measurements are not directly biased by telluric water. Variations in the water column, however, may indirectly affect observations by manifesting as additional noise in our light curves. Due to the narrow width of the water line $(\sim 0.03 \mathrm{~nm}$ FWHM; Allart et al. 2018, 2019; Nortmann et al. 2018; Salz et al. 2018; Alonso-Floriano et al. 2019), relative to the filter $(0.635 \mathrm{~nm}$ FWHM), variations would need to be large $(\sim 10 \%)$ on timescales comparable to our exposure times $(\sim 1$ minute) to manifest above the photometric noise as extra white noise. Smaller variations over long timescales could manifest as a time-correlated trend in our photometric data. If warranted by the data in the future, we could correct such time-correlated variations with a Gaussian process, but we see little evidence of this effect in our final light curves.

To correct for telluric $\mathrm{OH}$ emission in each image, we median-scaled our sky background frame to the sigma-clipped science data in 10 pixel steps radially outwards from the filter zero-point (where, as in Equation (1) above, the pixel scale is 0 .!25). This procedure removed a majority of the telluric background as shown in Figure 2, but in some images left a small amount of residual local structure with maximum amplitude of $10 \mathrm{ADU} / \mathrm{pixel}$, perhaps due to spatial variation of $\mathrm{OH}$ emission on the sky. Because even these residuals were locally quite stable, we estimated and removed the remaining background during aperture photometry using an annular region around each source as described below. This local background varies quite slowly in time and we find that this procedure reliably eliminates time-correlated noise from sky background and tellurics.

\subsubsection{Aperture Photometry}

We detected and registered the positions of the target and comparison stars using Aladin Lite (Bonnarel et al. 2000; Boch \& Fernique 2014) as described in Vissapragada et al. (2020). For both WASP-69 and WASP-52, we registered four comparison stars in addition to the target; for WASP-69, the target and comparison stars are visible in the backgroundcorrected image in Figure 2. We performed aperture photometry on each source in each image with the photutils package (Bradley et al. 2016) where we stepped through a range of circular apertures (from 7 to 15 pixels in radius in one pixel steps). The positions of the aperture centers were allowed to shift to trace telescope pointing drift. For WASP-69 and associated comparison stars, these varied by less than 2 pixels over most of the night, but a guiding error compromised the last hour of data collection. Excluding this last hour did not change our final answers but substantially decreased the correlated noise, so we choose to exclude these images from the final photometry. For WASP-52 we encountered a guiding jump of about 6 pixels an hour from the start of the observation, and again an hour from the end of the observation. These jumps were purely in the R.A. direction and are thus likely related to a known issue with the R.A. guiding on the telescope. Including the data marred by guiding errors substantially increased the correlated noise in the final light curve, so we opted to leave them out for our analysis of WASP-52b.

We estimated the residual local background by measuring the sigma-clipped median for an annulus around each source with an inner radius of 25 pixels and an outer radius of 50 pixels. We then trimmed outliers in the raw light curves using the moving median procedure from Vissapragada et al. (2020). We determined the optimal photometric aperture size by minimizing the rms of the residuals after the light-curve modeling described in the next section. Our optimal apertures were 10 and 8 pixels in radius for WASP-69 and WASP-52, respectively. A zoomed-in view of WASP-69 with fluxweighted centroid, best aperture, and background annulus overplotted is shown in Figure 2. It is clear from this figure that a 10 pixel aperture misses some flux from the target star. However, when the aperture size increases to encompass all of the flux from the target star, the comparison star light curves decrease in quality due to increased noise from the sky background. We tested the impact of using different aperture sizes for each source and found that this sharply degraded the quality of the final light curve, likely because PSF changes due to seeing variations impact each aperture differently. We therefore chose to continue with the selected optimal apertures in our final light-curve modeling. Raw light curves in the optimized apertures are given in Figure 3 for both planets.

\subsection{Light-curve Modeling}

We modeled the light curves with a procedure similar to that used in Vissapragada et al. (2020), which we briefly summarize here for completeness. Each target light curve is modeled as a transit light-curve model (which is computed with batman; Kreidberg 2015) multiplied by a systematics model. The systematics are further modeled as a linear trend in time plus a linear combination of the comparison star light curves, with new best-fitting linear coefficients chosen every time the transit light curve is modified. As in Vissapragada et al. (2020), our six fit parameters were the transit depth $\left(R_{\mathrm{p}} / R_{\star}\right)^{2}$, a timing offset from the predicted midtransit time $\Delta t_{0}$, a linear trend in time $\alpha$, the inclination $i$, the scaled semimajor axis $a / R_{\star}$, and a parameter describing the photometric scatter in excess of shot noise $\log \left(\sigma_{\text {extra }}\right)$. The excess scatter that we calculate is added in quadrature to the photometric error bars on each data point to give the final errors. We calculated custom quadratic limb darkening coefficients $u_{1}$ and $u_{2}$ in our bandpass using $1 \mathrm{dtk}$ (Husser et al. 2013; Parviainen \& Aigrain 2015) and the stellar parameters from Anderson et al. (2014) and Hébrard et al. (2013) for WASP-69 and WASP-52, respectively. These coefficients are reported in Table 1 . We additionally explored the possibility of fitting the quadratic limb darkening coefficients using the triangular sampling algorithm from Kipping (2013), but found that this did not make a substantive difference in our final results, so we chose to leave these coefficients fixed.

We first fit the data using the Powell minimizer from scipy (Virtanen et al. 2019), and we use this initial solution as a starting point for a Markov Chain Monte Carlo investigation 


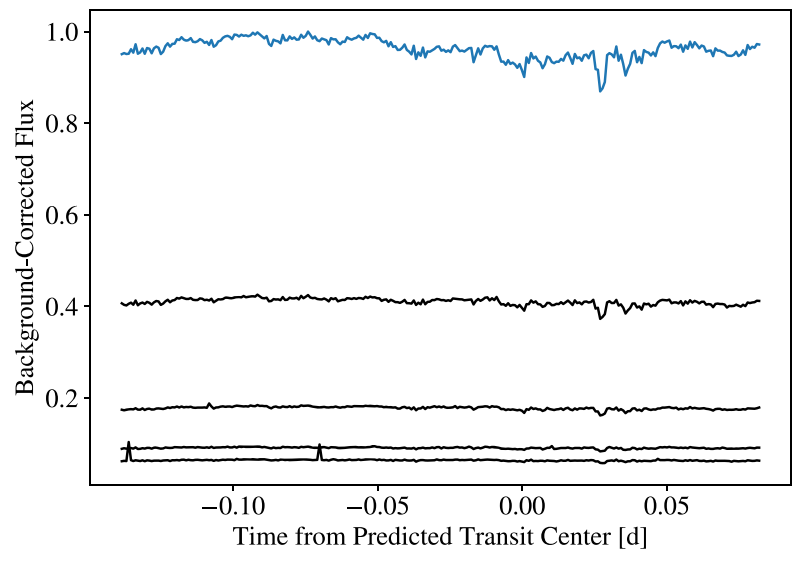

(a)

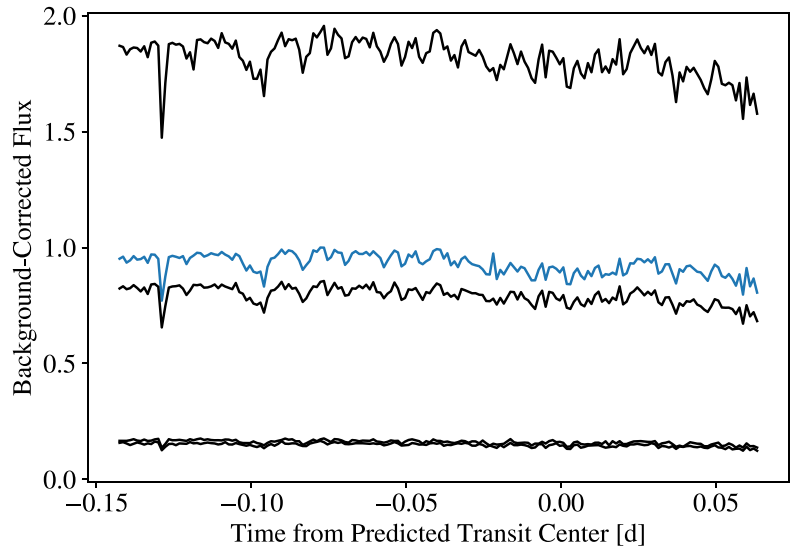

(b)

Figure 3. Raw light curves for stars in the WASP-69 field (a) and WASP-52 field (b). In both plots, the target light curve is shown in blue, comparison light curves are shown in black, and all light curves have been normalized to the target light-curve maximum.

Table 1

Light-curve Fitting Results

\begin{tabular}{|c|c|c|c|c|c|}
\hline \multirow{2}{*}{ Parameter } & \multicolumn{2}{|c|}{ Prior } & \multicolumn{2}{|c|}{ Posterior } & \multirow{2}{*}{ Note } \\
\hline & WASP-69b & WASP-52b & WASP-69b & WASP-52b & \\
\hline$P$ (days) & 3.86814098 & 1.74978179 & (fixed) & (fixed) & $(1),(2)$ \\
\hline$u_{1}$ & 0.3975 & 0.3635 & (fixed) & (fixed) & $(3),(4),(5)$ \\
\hline$u_{2}$ & 0.1156 & 0.1229 & (fixed) & (fixed) & $(3),(4),(5)$ \\
\hline$e$ & 0. & 0. & (fixed) & (fixed) & $(4),(5)$ \\
\hline$i\left(^{\circ}\right)$ & $\mathcal{N}(86.71,0.20)$ & $\mathcal{N}(85.17,0.13)$ & $86.63_{-0.15}^{+0.15}$ & $85.20_{-0.12}^{+0.12}$ & $(4),(6)$ \\
\hline$a / R_{\star}$ & $\mathcal{N}(12.00,0.46)$ & $\mathcal{N}(7.22,0.07)$ & $11.82_{-0.25}^{+0.25}$ & $7.207_{-0.062}^{+0.062}$ & $(4),(6)$ \\
\hline$\alpha$ & $\mathcal{U}(-0.2,0.2)$ & $\mathcal{U}(-0.2,0.2)$ & $0.0160_{-0.0025}^{+0.0026}$ & $0.0811_{-0.0012}^{+0.0012}$ & $\ldots$ \\
\hline $\log \left(\sigma_{\text {extra }}\right)$ & $\mathcal{U}(-3.5,-2.0)$ & $\mathcal{U}(-3.5,-2.0)$ & $-2.711_{-0.025}^{+0.025}$ & $-2.422_{-0.070}^{+0.006}$ & $\ldots$ \\
\hline
\end{tabular}

Note. (1) WASP-69b ephemerides from Baştürk et al. (2019); (2) WASP-52b ephemerides from Baluev et al. (2019); (3) quadratic limb darkening coefficients calculated with 1 dtk (Husser et al. 2013; Parviainen \& Aigrain 2015); (4) stellar parameters (for limb darkening calculations), $e, i$, and $a / R_{\star}$ from Anderson et al. (2014) for WASP-69b; (5) stellar parameters (for limb darkening calculations) and $e$ from Hébrard et al. (2013) for WASP-52b; (6) $i$ and $a / R_{\star}$ from Alam et al. (2018) for WASP-52b. Note also that $\mathcal{N}(a, b)$ denotes a Gaussian distribution centered on $a$ with standard deviation $b$, and $\mathcal{U}(a, b)$ denotes a uniform distribution between $a$ and $b$.

with emcee (Foreman-Mackey et al. 2013). We run 50 chains for $10^{3}$ steps to burn in, and then $10^{4}$ steps (which corresponds to at least 150 integrated autocorrelation times for each parameter) for the actual run. The posteriors from these lightcurve fits are summarized in Table 1 , and they are visualized in the Appendix.

\section{Results and Discussion}

\subsection{WASP-69b}

Our helium light curve for WASP-69b, along with a best-fit model, residuals, and an Allan deviation plot for the residuals are shown in Figure 4(a), and a corner plot summarizing the fit posteriors is shown in Figure A1. We measure a transit depth of $2.152 \% \pm 0.045 \%$. As a reference value, we use the HST WFC3 spectrum obtained by Tsiaras et al. (2018), who report an average transit depth of $1.6538 \% \pm 0.0045 \%$ between 1110.8 and $1141.6 \mathrm{~nm}$. Our transit depth exceeds the reference value by $11.1 \sigma$, indicating a secure detection of helium in the atmosphere of WASP-69b. We prefer a transit timing solution slightly earlier than, but not incompatible with, the ephemeris from Baştürk et al. (2019). Our constraints on $i$ and $a / R_{\star}$ are compatible with those from Anderson et al. (2014). We note, however, slight covariances between these parameters and the transit depth in Figure A1. Updated knowledge on these parameters may allow us to better constrain the transit depth in the future.

We achieved a per-point rms of $8.21 \mathrm{ppm} / \mathrm{pt}$ across 271 points. The final scatter in our residuals was $2.0 \times$ the shot noise (the noise floor set by Poisson statistics on our total detected photon counts, of which approximately $25 \%$ are background counts due to $\mathrm{OH}$ emission). A small correlated component to the noise appears on 10 minute timescales (see Figure 4(a)); we obtain a Carter \& Winn (2009) $\beta$ factor of 1.08. This is noticeably larger scatter (relative to shot noise) than what we have typically achieved in the past for targets of 

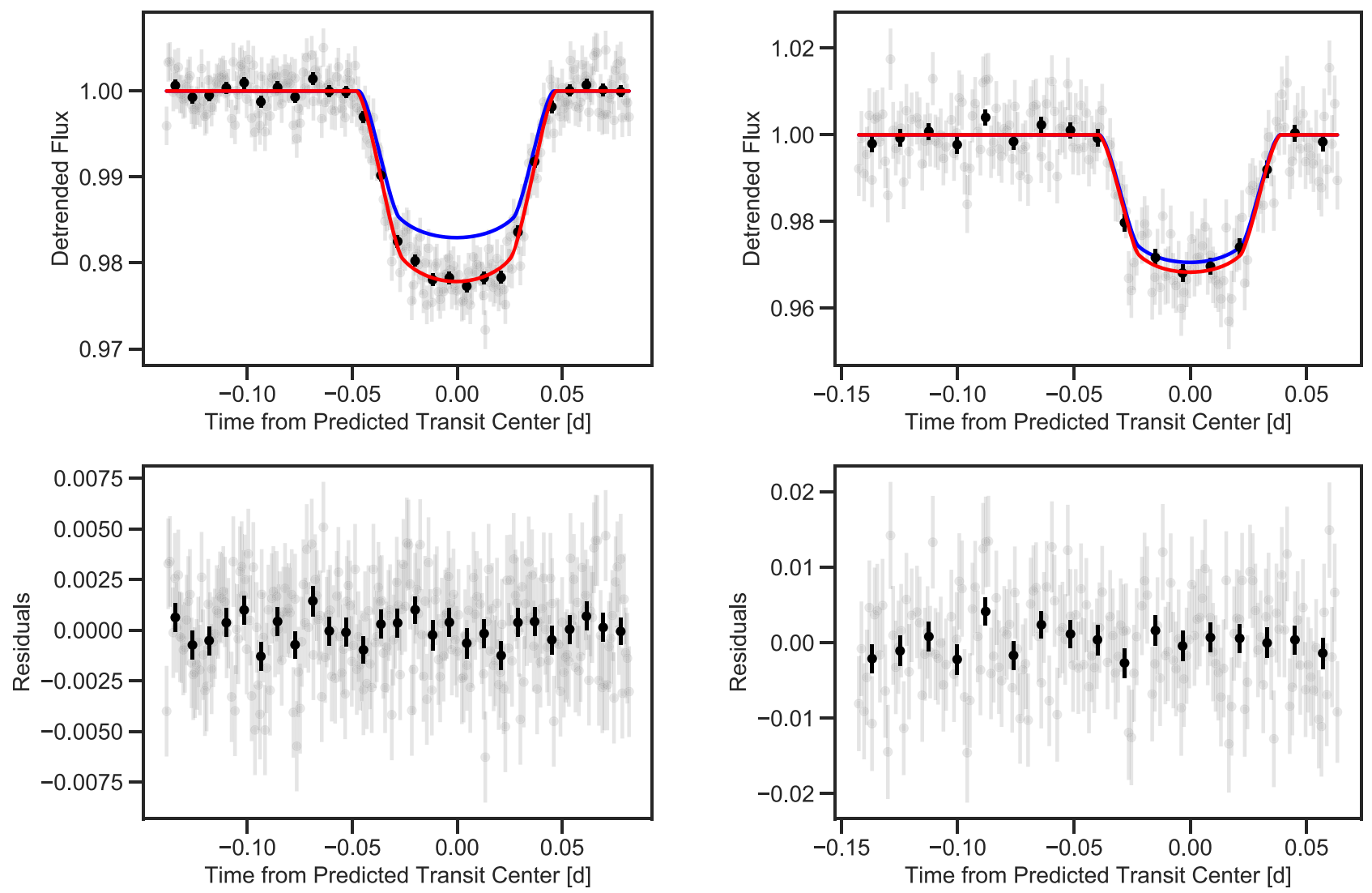

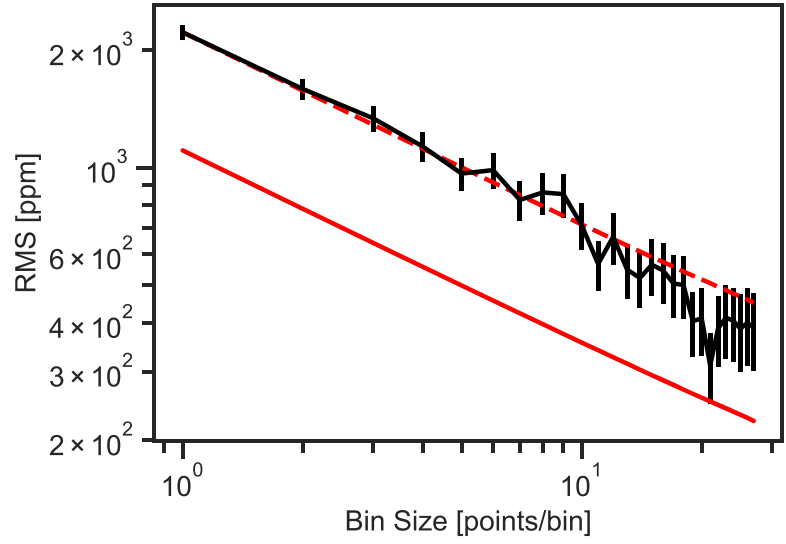

(a)

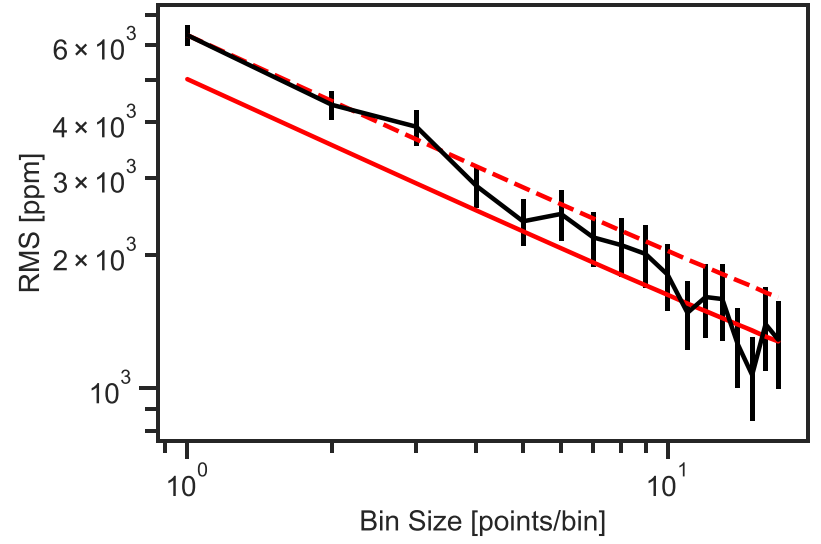

(b)

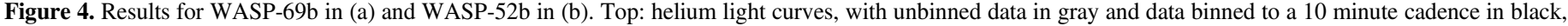

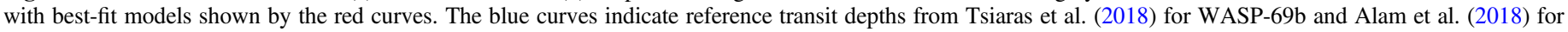

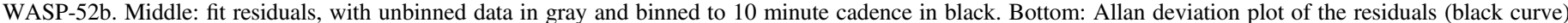

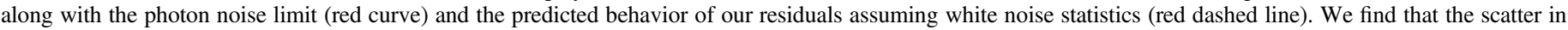
these data is $2.0 \times$ the photon noise limit for WASP-69b and $1.3 \times$ the photon noise limit for WASP-52b.

(The data used to create this figure are available.)

similar apparent brightness (Vissapragada et al. 2020). We observed this target at high efficiency (collecting light $87.6 \%$ of the time we were on sky), and the long exposure times make scintillation noise an unlikely culprit (Stefansson et al. 2017). This may be a signature of variation in the stellar He I line itself (Sanz-Forcada \& Dupree 2008; Andretta et al. 2017; Salz et al. 2018), but if such variations occur on long timescales (e.g., from spots on the stellar surface), then they would be corrected by our linear detrending model, and if they occur on short timescales, they would manifest as strong red noise in the light curve, which we do not observe. Rather, the likely explanation for our photometric performance is a paucity of good comparison stars in the field. WASP-69 inhabits a fairly sparse field already, and to compound the issue we are limited in target placement to the arc shown in Figure 1(d), which may put otherwise accessible comparison stars outside the field of 


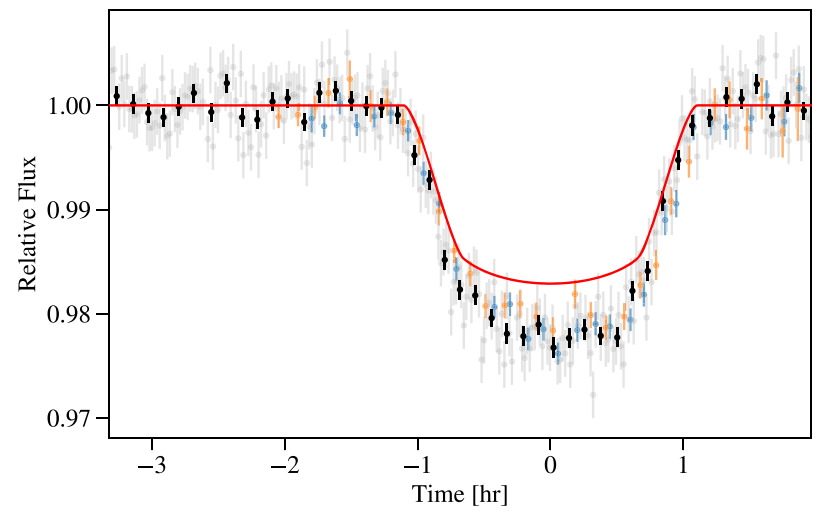

(a)

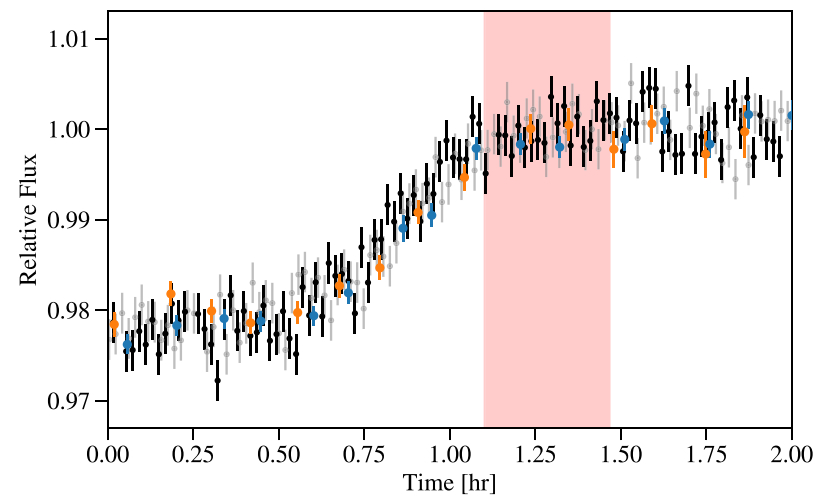

(b)

Figure 5. (a) WIRC light curve of WASP-69b (unbinned in gray and binned to 7 minute cadence in black) compared to CARMENES light curves (computed by integrating CARMENES spectra against our transmission function) from Nortmann et al. (2018) in blue and orange (their first and second nights of data collections, respectively). The comparison light curve from Tsiaras et al. (2018) is shown in red. (b) Mirrored, unbinned WIRC light curve, with ingress shown in gray and egress shown in black. Data from CARMENES are again shown in blue and orange for the first and second nights of data collection (Nortmann et al. 2018). The postegress absorption reported by Nortmann et al. (2018) would fall within the red region. We do not see significant evidence for it here, but the asymmetry is also washed out in the calculated CARMENES light curve due to our wide bandpass (relative to the CARMENES resolution element).

view. Thus, we are limited in our ability to obtain many good comparison stars for this technique, which here is likely the ultimate limiting factor in our photometry.

We now assess how our transit measurement compares to the spectroscopic measurement of Nortmann et al. (2018). We took their reduced stellar spectra gathered over two nights of observation and converted these from the planet rest frame (in which the reduced data were provided) back to the telluric rest frame. For each spectrum (which we label $f_{i, \lambda}$, where $i$ indexes time and $\lambda$ indexes wavelength), we calculated the excess absorption signal $f_{i}$ in our bandpass using our measured transmission function $T_{\lambda}$ via

$$
f_{i}=\frac{\int f_{i, \lambda} T_{\lambda} d \lambda}{\int T_{\lambda} d \lambda} .
$$

The timeseries $f$ then represents the excess absorption in the helium line during the transit as would be measured by CARMENES through our helium filter. To this we added the broadband light curve (calculated with the parameters of Tsiaras et al. 2018) which gave the total light curve as would have been observed by WIRC. We repeated this procedure for both nights of CARMENES data collection (with 35 spectra in night 1 and 31 spectra in night 2), and we present our results compared to the two CARMENES timeseries in Figure 5(a). Our data show good agreement with those collected by Nortmann et al. (2018).

Nortmann et al. (2018) also report the detection of an asymmetric transit in $\mathrm{He}$ I, with egress extending about half an hour past ingress. We do not find strong evidence for this effect in our light curve. In Figure 5(b), we show our WASP-69b light curve mirrored across our best-fit midtransit time; there is no visible absorption in the postegress window where Nortmann et al. (2018) report an extended tail. While we do not see strong evidence for this effect in our light curve, however, we cannot rule it out. The amplitude of the reported postegress absorption is of order $0.5 \%$; when diluted through our transmission function this becomes a $500 \mathrm{ppm}$ effect which we are not significantly sensitive to on a 22 minute timescale (our rms on this timescale is $388 \mathrm{ppm}$ ). Repeated observations of WASP-69b may allow us to constrain the transit asymmetry in the future.

\subsection{WASP-52b}

Our helium light curve for WASP-52b, along with a best-fit model, residuals, and an Allan deviation plot for the residuals are shown in Figure 4(b), and a corner plot summarizing the fit posteriors is shown in Figure A2. We measure a transit depth of $2.97_{-0.13}^{+0.13} \%$, which exceeds the spot-uncorrected transit depth between 898.5 and $1030.0 \mathrm{~nm}(2.76 \% \pm 0.021 \%)$ from Alam et al. (2018) by $1.6 \sigma$. Assuming the same line structure shape as is observed for WASP-69b (Nortmann et al. 2018), this converts to an amplitude of $1.31 \% \pm 0.94 \%$ in the deepest line of the triplet. This is meant only to give a sense of what one might expect at high resolution; in reality, line shapes can vary from planet to planet, and there is no guarantee that assuming the line shape of WASP-69b is correct (Allart et al. 2018, 2019; Nortmann et al. 2018; Salz et al. 2018; Alonso-Floriano et al. 2019; Kirk et al. 2020). We obtained a per-point rms of $35.6 \mathrm{ppm} / \mathrm{pt}$ across 177 points. The scatter in the light curve was $1.3 \times$ the photon noise limit, binning down like white noise (see the bottom panel of Figure 4). This performance is comparable to what we have achieved in the past for similar targets (Vissapragada et al. 2020), despite the fact that there were only four comparison stars in the field of view.

WASP-52 is a young $\left(0.4_{-0.2}^{+0.3} \mathrm{Gyr}\right)$, active host star, with a $\log R^{\prime}{ }_{\mathrm{HK}}$ index of $-4.4 \pm 0.2$ (Hébrard et al. 2013), and many authors have observed and analyzed the effects of spots and plages (Kirk et al. 2016; Chen et al. 2017; Louden et al. 2017; Mancini et al. 2017; Alam et al. 2018; Bruno et al. 2018, 2020; May et al. 2018). Considering the proposed relationship between planetary metastable helium absorption and stellar activity (Nortmann et al. 2018; Alonso-Floriano et al. 2019), WASP-52 remains a high-priority target for future work. Follow-up observations with high-resolution spectroscopic facilities on larger telescopes should be able to detect absorption and quantify the line shape (which we must assume here) for this rather challenging target. We note that confident detections of $\mathrm{Na}, \mathrm{K}$, and $\mathrm{H} \alpha$ absorption in the atmosphere of this planet recently required three transits with the ESPRESSO 


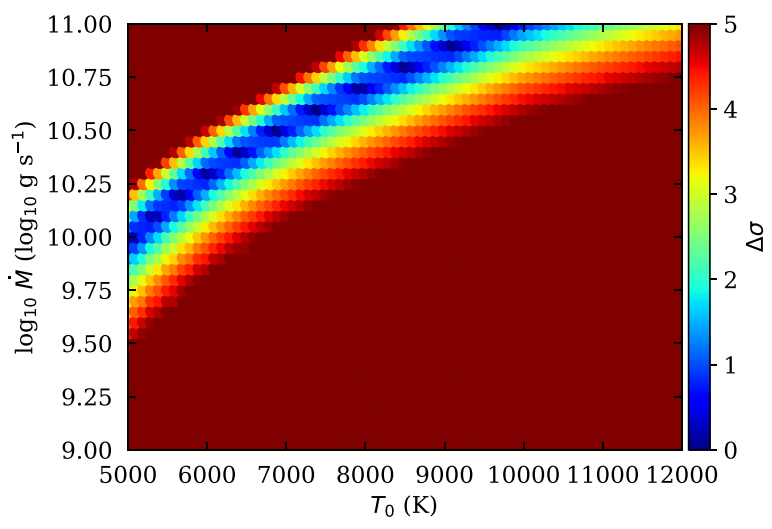

(a)

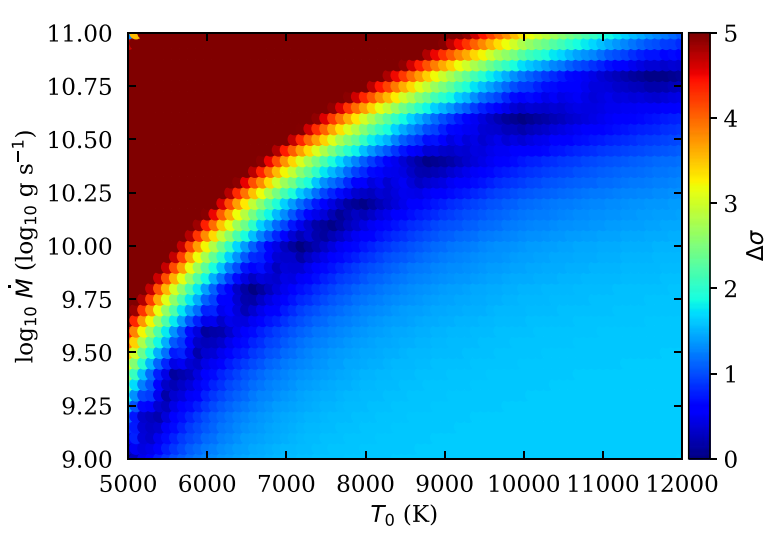

(b)

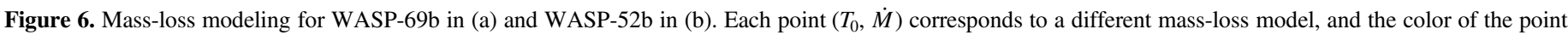
indicates the $\sigma$ discrepancy between that model and the data presented in Figure 5.

high-resolution spectrograph on the Very Large Telescope (Chen et al. 2020). Though its host star is relatively faint, WASP-52b is well worth additional observations in metastable helium, as the other detected atomic species will provide some context for modeling the upper atmosphere of this planet.

\subsection{Mass-loss Modeling}

We interpret our observations of WASP-69b and WASP-52b using the Oklopčić \& Hirata (2018) model. Despite our lack of a significant detection for WASP-52b, we model potential outflows from this planet to set an upper limit on the mass-loss rate corresponding to our upper limit on the excess absorption. As WASP-52b is a high-priority target for future observations (Kirk et al. 2020), this is a particularly important constraint that we can obtain from our light curve.

We first computed grids of atmospheric mass-loss models; following Oklopčić \& Hirata (2018) and Mansfield et al. (2018), we computed 1D density and velocity profiles for a 90\%-10\% hydrogen-helium atmosphere losing mass to an isothermal Parker wind. These profiles spanned $5000 \mathrm{~K}-$ $12,000 \mathrm{~K}$ in thermosphere temperature $T_{0}$ and $10^{9}-10^{11} \mathrm{~g} \mathrm{~s}^{-1}$ in mass-loss rate $\dot{M}$, with the ranges motivated by hydrodynamics simulations of atmospheric escape (Salz et al. 2016). Level populations for hydrogen and helium were then computed for each profile. As there are no measurements of the stellar UV spectra (required for computing photoionization rates) for WASP-69 and WASP-52, we used UV spectra from MUSCLES (France et al. 2016) of stars with similar spectral type. For WASP-69, we used HD 85512 (K6) and for WASP52 we used $\epsilon$ Eri (K2).

The resulting density profiles of $2^{3} \mathrm{~S}$ He were then used to compute the transit depth in the line given our filter transmission function, and the model transit depths were compared to those that we report in Table 1 . We opted to compare only the transit depths from the outflow models to our data rather than the full light curve, as the full computation is substantially more expensive for a marginal gain in accuracy for the model comparison (relative to our photometric uncertainties). In Figure 6, we show how the model grids compare to our data, parameterized by the number of standard deviations away from our data. For WASP-69b we obtain a curved contour of best-fit solutions, indicating a known degeneracy between mass-loss rate and thermosphere temperature due to our inability to resolve line shapes (Mansfield et al. 2018).

To summarize the contours in Figure 6, we quote our constraints on the mass-loss rate at two possible thermosphere temperatures. At $T_{0}=7000 \mathrm{~K}(12,000 \mathrm{~K})$ we obtain a corresponding mass-loss rate

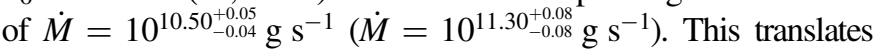
to $5.25_{-0.46}^{+0.65} \times 10^{-4} M_{\mathrm{J}} \mathrm{Gyr}^{-1} \quad\left(3.32_{-0.56}^{+0.67} \times 10^{-3} M_{\mathrm{J}} \mathrm{Gyr}^{-1}\right)$. The mass-loss rate for WASP-69b is therefore very similar to those reported for HAT-P-11b and WASP-107b (Allart et al. 2018, 2019; Mansfield et al. 2018; Spake et al. 2018; Kirk et al. 2020), which should be typical for planets at similar distances and gravitational potentials (Salz et al. 2016). For WASP-52b, we can set a 95th percentile upper limit of $\dot{M}<10^{10.1} \mathrm{~g} \mathrm{~s}^{-1}\left(10^{11.1} \mathrm{~g} \mathrm{~s}^{-1}\right)$ at $T_{0}=7000 \mathrm{~K}(12,000 \mathrm{~K})$. This translates to $2.1 \times$ $10^{-4} M_{\mathrm{J}} \mathrm{Gyr}^{-1}\left(2.1 \times 10^{-3} M_{\mathrm{J}} \mathrm{Gyr}^{-1}\right)$. We conclude from these measurements that, barring substantial changes in orbital distance and stellar irradiation, WASP-69b $\left(M_{\mathrm{p}}=0.26 M_{\mathrm{J}}\right)$ and WASP-52b $\left(M_{\mathrm{p}}=0.46 M_{\mathrm{J}}\right)$ will survive over the lifetime of their host stars (losing at most a few percent in envelope mass), and their compositions will not be substantially impacted by mass loss.

\section{Conclusions}

In this work, we have presented a new photometric technique to observe the metastable $2^{3} \mathrm{~S}$ helium absorption feature near $1083.3 \mathrm{~nm}$ using an ultranarrowband filter and a beam-shaping diffuser. We benchmarked this new technique by observing WASP-69b, a planet for which the shape of the helium feature has been measured with high-resolution spectroscopy (Nortmann et al. 2018). Our technique detects helium absorption to $11.1 \sigma$ confidence (a single-transit signal-to-noise ratio comparable to that achieved with CARMENES) in this planet's atmosphere, at a level consistent with previous observations. Additionally, for WASP-52b we set a 95th percentile upper limit on excess absorption in the helium bandpass of $0.47 \%$. We find that the quality of our photometry relative to the photon noise limit depends sensitively on the availability of comparison sources. Interpreting our results with atmospheric mass-loss modeling allows us to constrain the mass-loss rate for WASP-69b to $5.25_{-0.46}^{+0.65} \times 10^{-4} M_{\mathrm{J}} \mathrm{Gyr}^{-1} \quad\left(3.32_{-0.56}^{+0.67} \times 10^{-3} M_{\mathrm{J}} \mathrm{Gyr}^{-1}\right)$ at $7000 \mathrm{~K}(12,000 \mathrm{~K})$, and additionally we set an upper limit to the mass-loss rate for WASP-52b at these temperatures of $2.1 \times 10^{-4} M_{\mathrm{J}} \mathrm{Gyr}^{-1}\left(2.1 \times 10^{-3} M_{\mathrm{J}} \mathrm{Gyr}^{-1}\right)$. These values are 
typical for other gaseous planets at similar gravitational potentials and orbital periods, and we conclude that both of these planets' atmospheres will not be substantially affected by mass loss for many billions of years.

Diffuser-assisted, ultranarrowband photometry on a widefield camera is a unique way to study exoplanet atmospheres, but it also comes with challenges. For the experimental setup detailed here, we sometimes have to settle for suboptimal photometry on brighter targets because we are observing in sparse fields with relatively few suitable comparison stars, and also because of the constraints imposed by the AOI shift effect. Additionally, the lack of a comparison bandpass means that we must rely on high-precision infrared transit measurements taken by other groups (or simultaneous measurements with different instruments) to establish the magnitude of the excess absorption in the helium line, rather than doing so in our own experimental setup. Both of these challenges could be overcome with photometers like those presented in Baker et al. (2019), which allow for simultaneous photometry of a target star in two adjacent passbands. Though our restricted instrumental setup does not presently allow us to use this method, or other multicolor imaging methods requiring dichroics (e.g., Dhillon et al. 2016), we believe these are fruitful avenues for future exploration in the context of narrow atomic and molecular features.

Despite the challenges we have encountered in our constrained experimental setup with WIRC, we have demonstrated that our system is capable of measuring mass-loss rates for most advantageous targets. Our technique occupies a unique niche in the current suite of approaches to metastable helium observations. First, the narrowband filter affords us better precision than space-based spectroscopy with HST WFC3, scaling from the precisions of Spake et al. (2018) and Mansfield et al. (2018). Second, while the James Webb Space Telescope will achieve much better precision (Allart et al. 2018), we can schedule and observe targets more readily on a ground-based $5 \mathrm{~m}$ telescope, allowing us to survey a wider range of planets. Third, the high efficiency of our technique lets us observe targets beyond the magnitude limits of highresolution spectrographs on smaller telescopes. With future WIRC observations, we aim to characterize the fundamental relationships between mass loss, stellar activity, high-energy flux, and planetary age (Nortmann et al. 2018; Alonso-Floriano et al. 2019; Oklopčić 2019; Owen 2019).
We thank the referee for a very thorough review that improved the quality of this work. We are grateful for the support of the Heising-Simons Foundation, which allowed us to purchase the narrowband filter used in this study. We thank Lisa Nortmann for providing us with the CARMENES data for WASP-69b. We recognize the Palomar Observatory staff for their support of our work, especially Paul Nied and Kajse Peffer for telescope operation and James Brugger, Greg Van Idsinga, Ernie Velador, and Brian Faull for assistance with helium lamp hardware. We also thank James Owen, Yanqin Wu, Trevor David, Ignas Snellen, Yayaati Chachan, Fei Dai, Munazza Alam, Nikolay Nikolov, Chaz Shapiro, Jennifer Milburn, Andy Boden, Roger Smith, and Keith Matthews for very useful conversations. S.V. is supported by an NSF Graduate Research Fellowship and the Paul \& Daisy Soros Fellowship for New Americans. H.A.K. acknowledges support from NSF CAREER grant 1555095 and NASA Origins grant NNX14AD22G. C.K.H. acknowledges support from the University of Maryland Department of Astronomy Honors Program, and from the Smithsonian Astrophysical Observatory REU program, which is funded in part by the National Science Foundation REU and Department of Defense ASSURE programs under NSF Grant no. AST-1852268, and by the Smithsonian Institution. A.O. acknowledges support by NASA through the NASA Hubble Fellowship grant HST-HF251443.001-A awarded by the Space Telescope Science Institute, which is operated by the Association of Universities for Research in Astronomy, Incorporated, under NASA contract NAS5-26555.

Facilities: Hale (WIRC), ADS, Exoplanet Archive.

Software: photutils (Bradley et al. 2016), numpy (van der Walt et al. 2011), astropy (Astropy Collaboration et al. 2013, 2018), scipy (Virtanen et al. 2019), matplotlib (Hunter 2007), batman (Kreidberg 2015), emcee (Foreman-Mackey et al. 2013), corner (Foreman-Mackey 2016), ldtk (Husser et al. 2013; Parviainen \& Aigrain 2015), Aladin Lite (Bonnarel et al. 2000; Boch \& Fernique 2014).

\section{Appendix Posterior Probability Distributions}

In this section, we show the posterior probability distributions for our light-curve fits to WASP-69b and WASP-52b, in Figures A1 and A2, respectively. 

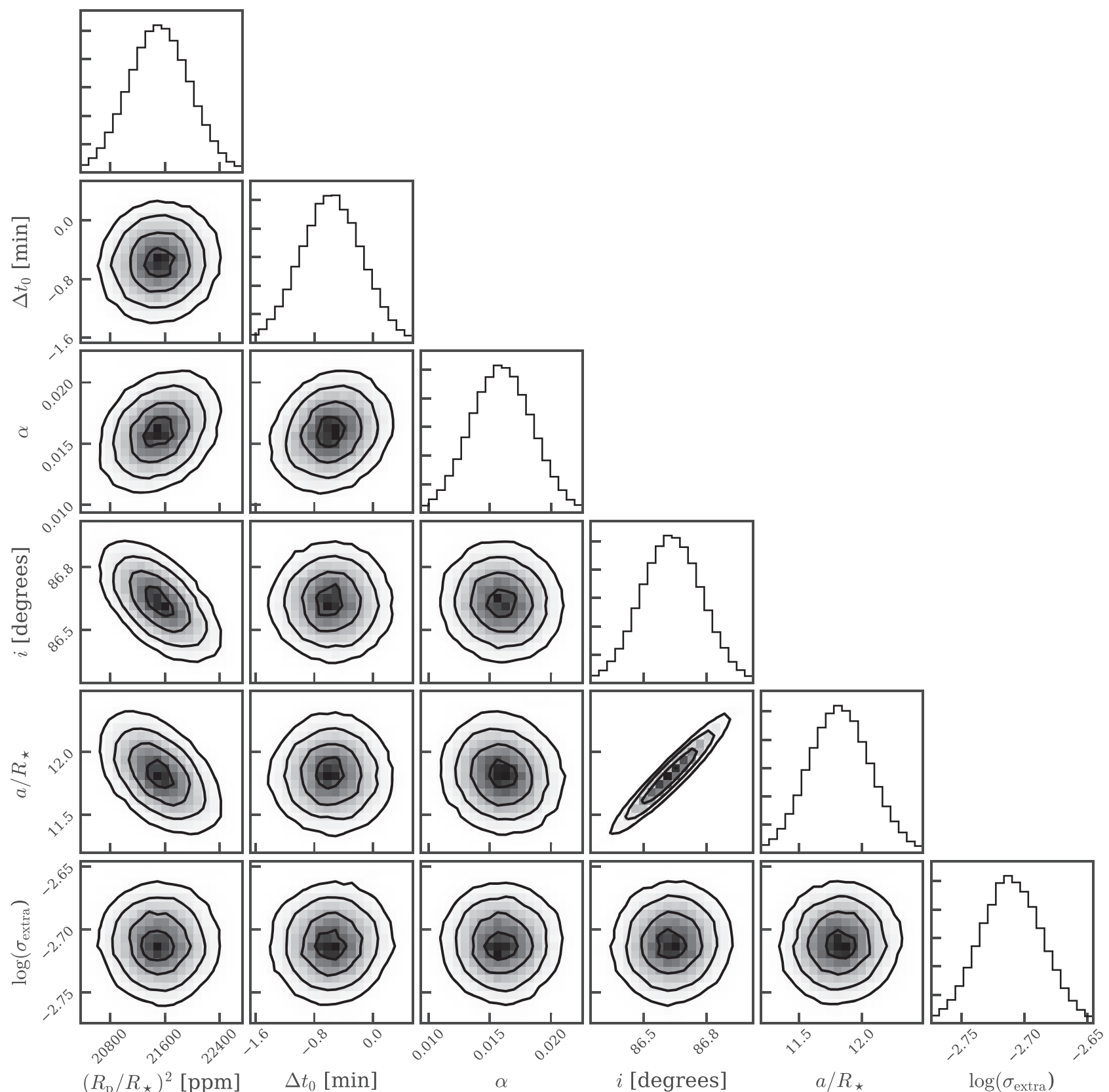

Figure A1. Corner plot of the posterior probability distributions for our fit to WASP-69b. The middle $99 \%$ of samples are shown with contours denoting 1,2 , and $3 \sigma$ boundaries. 


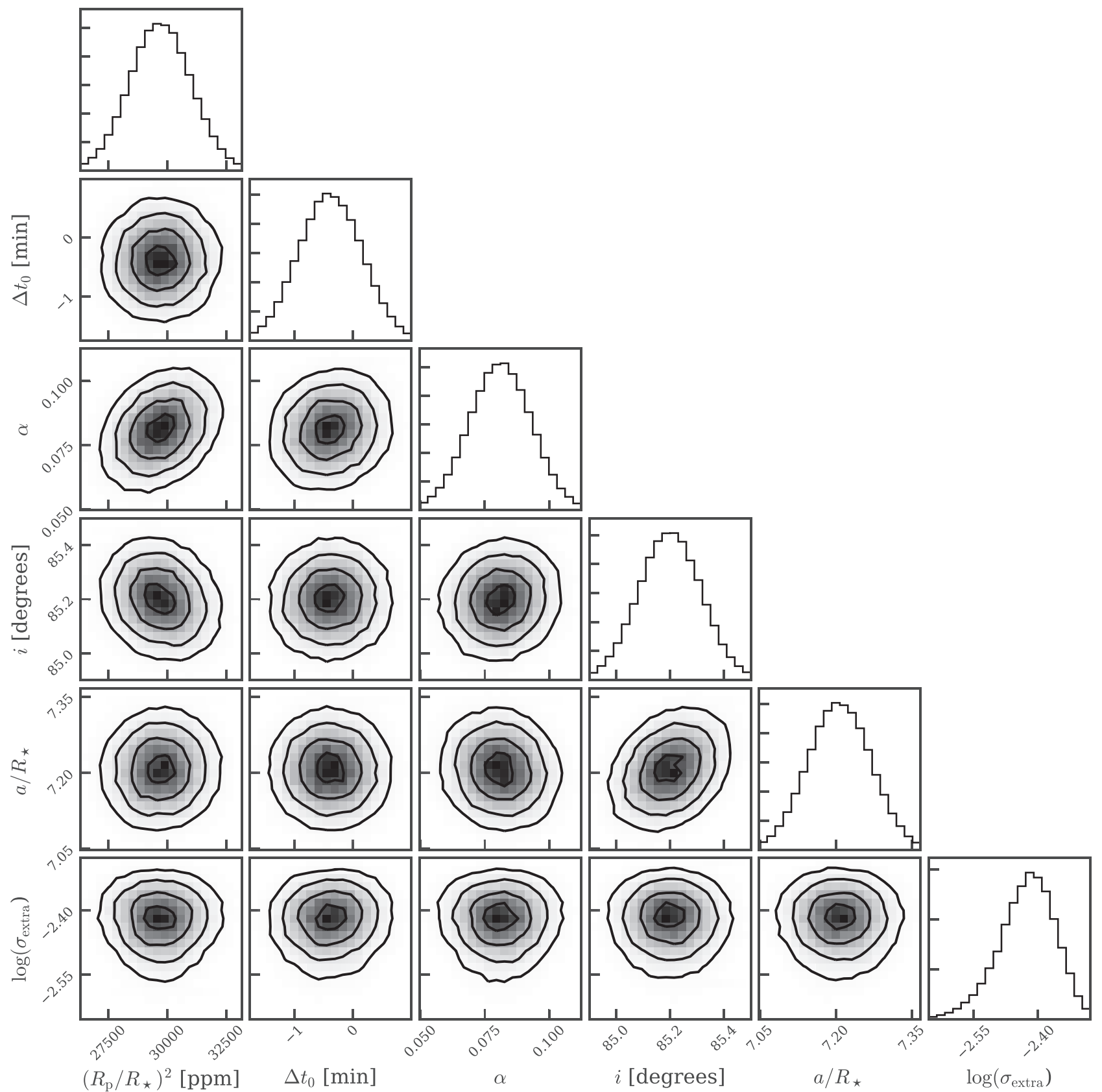

Figure A2. Same as Figure A1 but for WASP-52b.

\section{ORCID iDs}

Shreyas Vissapragada (1) https://orcid.org/0000-0003-2527-1475 Nemanja Jovanovic 으 https://orcid.org/0000-0001-5213-6207 Caleb K. Harada (1) https://orcid.org/0000-0001-5737-1687 Antonija Oklopčić iㅣ https://orcid.org/0000-0002-9584-6476 Dimitri Mawet (i) https://orcid.org/0000-0002-8895-4735 Maxwell A. Millar-Blanchaer (1) https://orcid.org/0000-00016205-9233

Samaporn Tinyanont (16 https://orcid.org/0000-0002-1481-4676 Gautam Vasisht (i) https://orcid.org/0000-0002-1871-6264

\section{References}

Alam, M. K., Nikolov, N., López-Morales, M., et al. 2018, AJ, 156, 298 Allart, R., Bourrier, V., Lovis, C., et al. 2018, Sci, 362, 1384
Allart, R., Bourrier, V., Lovis, C., et al. 2019, A\&A, 623, A58

Alonso-Floriano, F. J., Snellen, I. A. G., Czesla, S., et al. 2019, A\&A, 629, A110

Anderson, D. R., Collier Cameron, A., Delrez, L., et al. 2014, MNRAS, 445, 1114

Andretta, V., Giampapa, M. S., Covino, E., et al. 2017, ApJ, 839, 97

Astropy Collaboration, Price-Whelan, A. M., Sipőcz, B. M., et al. 2018, AJ, 156,123

Astropy Collaboration, Robitaille, T. P., Tollerud, E. J., et al. 2013, A\&A, 558, A33

Baker, A. D., Blake, C. H., \& Halverson, S. 2019, PASP, 131, 064402

Baluev, R. V., Sokov, E. N., Jones, H. R. A., et al. 2019, MNRAS, 490, 1294

Baştürk, Ö, Esmer, E. M., Torun, S, et al. 2019, in AIP Conf. Proc. 2178 Turkish Physical Society 35th International Physics Congress (TPS35), ed. B. Akkuş et al. (Melville, NY: AIP), 030019

Bernath, P. F., \& Colin, R. 2009, JMoSp, 257, 20

Boch, T., \& Fernique, P. 2014, in ASP Conf. Ser. 485, Astronomical Data Analysis Software and Systems XXIII, ed. N. Manset \& P. Forshay (San Fransisco, CA: ASP), 277 
Bonnarel, F., Fernique, P., Bienaymé, O., et al. 2000, A\&AS, 143, 33 Bourrier, V., Ehrenreich, D., Allart, R., et al. 2017a, A\&A, 602, A106 Bourrier, V., Ehrenreich, D., King, G., et al. 2017b, A\&A, 597, A26 Bourrier, V., Ehrenreich, D., Wheatley, P. J., et al. 2017c, A\&A, 599, L3

Bourrier, V., Lecavelier des Etangs, A., Ehrenreich, D., et al. 2018, A\&A, 620, A147

Bradley, L., Sipocz, B., Robitaille, T., et al. 2016, Photutils: Photometry tools, version 0.7.1, Astrophysics Source Code Library, ascl:1609.011

Bruno, G., Lewis, N. K., Alam, M. K., et al. 2020, MNRAS, 491, 5361

Bruno, G., Lewis, N. K., Stevenson, K. B., et al. 2018, AJ, 156, 124

Carter, J. A., \& Winn, J. N. 2009, ApJ, 704, 51

Chen, G., Casasayas-Barris, N., Palle, E., et al. 2020, A\&A, 635, A171

Chen, G., Pallé, E., Nortmann, L., et al. 2017, A\&A, 600, L11

Cloutier, R., \& Menou, K. 2020, AJ, 159, 211

Crossfield, I. J. M., Barman, T., Hansen, B., et al. 2019, RNAAS, 3, 24

Dhillon, V. S., Marsh, T. R., Bezawada, N., et al. 2016, Proc. SPIE, 9908, 99080Y

dos Santos, L. A., Ehrenreich, D., Bourrier, V., et al. 2020, A\&A, 634, L4

Ehrenreich, D., Bourrier, V., Bonfils, X., et al. 2012, A\&A, 547, A18

Ehrenreich, D., Bourrier, V., Wheatley, P. J., et al. 2015, Natur, 522, 459

Foreman-Mackey, D. 2016, JOSS, 1, 24

Foreman-Mackey, D., Hogg, D. W., Lang, D., \& Goodman, J. 2013, PASP, 125,306

France, K., Loyd, R. O. P., Youngblood, A., et al. 2016, ApJ, 820, 89

Fulton, B. J., \& Petigura, E. A. 2018, AJ, 156, 264

Fulton, B. J., Petigura, E. A., Howard, A. W., et al. 2017, AJ, 154, 109

Gaidos, E., Hirano, T., Mann, A. W., et al. 2020, MNRAS, in press

García Muñoz, A., Youngblood, A., Fossati, L., et al. 2020, ApJL, 888, L21

Ghinassi, F., Licandro, J., Oliva, E., et al. 2002, A\&A, 386, 1157

Hardegree-Ullman, K. K., Zink, J. K., Christiansen, J. L., et al. 2020, ApJS, 247,28

Hébrard, G., Collier Cameron, A., Brown, D. J. A., et al. 2013, A\&A, 549, A134

Hunter, J. D. 2007, CSE, 9, 90

Husser, T.-O., Wende-von Berg, S., Dreizler, S., et al. 2013, A\&A, 553, A6

Indriolo, N., Hobbs, L. M., Hinkle, K. H., et al. 2009, ApJ, 703, 2131

Kipping, D. M. 2013, MNRAS, 435, 2152

Kirk, J., Alam, M. K., Lopez-Morales, M., \& Zeng, L. 2020, AJ, 159, 115

Kirk, J., Wheatley, P. J., Louden, T., et al. 2016, MNRAS, 463, 2922

Kreidberg, L. 2015, PASP, 127, 1161

Kreidberg, L., \& Oklopčić, A. 2018, RNAAS, 2, 44
Kulow, J. R., France, K., Linsky, J., et al. 2014, ApJ, 786, 132

Lavie, B., Ehrenreich, D., Bourrier, V., et al. 2017, A\&A, 605, L7

Lecavelier des Etangs, A., Bourrier, V., Wheatley, P. J., et al. 2012, A\&A, 543, L4

Lecavelier Des Etangs, A., Ehrenreich, D., Vidal-Madjar, A., et al. 2010, A\&A, 514, A72

Lopez, E. D., \& Fortney, J. J. 2013, ApJ, 776, 2

Louden, T., Wheatley, P. J., Irwin, P. G. J., et al. 2017, MNRAS, 470, 742

Mancini, L., Southworth, J., Raia, G., et al. 2017, MNRAS, 465, 843

Mansfield, M., Bean, J. L., Oklopčić, A., et al. 2018, ApJL, 868, L34

May, E. M., Zhao, M., Haidar, M., et al. 2018, AJ, 156, 122

Ninan, J. P., Stefansson, G., Mahadevan, S., et al. 2020, AJ, 894, 97

Nortmann, L., Pallé, E., Salz, M., et al. 2018, Sci, 362, 1388

Oklopčić, A. 2019, ApJ, 881, 133

Oklopčić, A., \& Hirata, C. M. 2018, ApJL, 855, L11

Oliva, E., Origlia, L., Scuderi, S., et al. 2015, A\&A, 581, A47

Owen, J. E. 2019, AREPS, 47, 67

Owen, J. E., \& Wu, Y. 2013, ApJ, 775, 105

Owen, J. E., \& Wu, Y. 2017, ApJ, 847, 29

Parviainen, H., \& Aigrain, S. 2015, MNRAS, 453, 3821

Salz, M., Czesla, S., Schneider, P. C., et al. 2016, A\&A, 586, A75

Salz, M., Czesla, S., Schneider, P. C., et al. 2018, A\&A, 620, A97

Sanz-Forcada, J., \& Dupree, A. K. 2008, A\&A, 488, 715

Seager, S., \& Sasselov, D. D. 2000, ApJ, 537, 916

Sing, D. K., Désert, J.-M., Fortney, J. J., et al. 2011, A\&A, 527, A73

Spake, J. J., Sing, D. K., Evans, T. M., et al. 2018, Natur, 557, 68

Stefansson, G., Mahadevan, S., Hebb, L., et al. 2017, ApJ, 848, 9

Tian, F. 2015, AREPS, 43, 459

Tinyanont, S., Millar-Blanchaer, M. A., Nilsson, R., et al. 2019, PASP, 131, 02500

Tsiaras, A., Waldmann, I. P., Zingales, T., et al. 2018, AJ, 155, 156

van der Walt, S., Colbert, S. C., \& Varoquaux, G. 2011, CSE, 13, 22

van Eylen, V., Agentoft, C., Lundkvist, M. S., et al. 2018, MNRAS, 479, 4786

Vidal-Madjar, A., Lecavelier des Etangs, A., Désert, J.-M., et al. 2003, Natur, 422, 143

Virtanen, P., Gommers, R., Oliphant, T. E., et al. 2019, arXiv:1907.10121

Vissapragada, S., Jontof-Hutter, D., Shporer, A., et al. 2020, AJ, 159, 108

Waalkes, W. C., Berta-Thompson, Z., Bourrier, V., et al. 2019, AJ, 158, 50

Wilson, J. C., Eikenberry, S. S., Henderson, C. P., et al. 2003, Proc. SPIE, 4841,451 